\title{
Molecular Design and Synthesis of Dicarbazolophane-Based Centro- symmetric through-Space Donors for Solution-Processed TADF OLEDs
}

\author{
Zhen Zhang, , Stefan Diesing, $\ddagger$ Ettore Crovini, Abhishek Kumar Gupta, Eduard Spuling, Xuemin Gan, \\ Olaf Fuhr, Martin Nieger, Zahid Hassan, Ifor D. W. Samuel, ${ }^{*}$ Stefan Bräse, ${ }^{*}$ and Eli Zysman-Colman*
}

\begin{abstract}
Conjugation-extended carbazolophane donors, dicarbazolophanes (DCCP), were designed and synthesized by a multi-fold stepwise Pd-catalyzed Buchwald-Hartwig amination/ring cyclization process. Further, elaboration of the DCCP core is possible with introduction of pendant carbazole derivative groups. This provided a way of tuning the optoelectronic properties of the thermally activated delayed fluorescence (TADF) compounds DCCPTRZtBu2, dtBuCzDCCPTRZtBu2, and dMeOCzDCCPTRZtBu 2 . Solution-processed OLEDs were fabricated and achieved maximum external quantum efficiencies $\left(\mathrm{EQE}_{\max }\right)$ of $8.2 \%$ and $\mathrm{EQE}$ of $7.9 \%$ at $100 \mathrm{~cd} / \mathrm{m}^{2}$.
\end{abstract}

The [2.2] paracyclophane (PCP) scaffolds have been used as platforms to study both planar chirality and through-space charge mobility and electronic communication in $\pi$-stacked molecular systems. ${ }^{1}$ The configurationally rigid structure PCP group is also photochemically stable, and chemically stable towards oxidation, acids and bases, thus making it a desirable molecular building block in organic electronics. ${ }^{2}$ We first reported the electron-donor carbazolophane (Czp), that merges the structure of the PCP with carbazole. ${ }^{3} \mathrm{Com}-$ pared to carbazole $(\mathrm{Cz})$, this donor adopts a more twisted conformation in donor-acceptor systems due to its larger size while the enlarged conjugation in the $\mathrm{CzP}$ results in a stronger electron donor. Further, the inherent planar chirality of the donor translates to emitters that show circularly polarized luminescence (CPL) (Figure 1). Incorporation of this donor produced the thermally activated delayed fluorescence (TADF) emitter CzpPhTRZ, which showed a small singlet-triplet energy splitting, $\Delta E_{\mathrm{ST}}$, of $0.16 \mathrm{eV}$ and a photoluminescence quantum yield, $\Phi_{\mathrm{PL}}$, of $70 \%$ in $10 \mathrm{wt} \%$ DPEPO doped film. The organic light-emitting diodes (OLEDs) showed a maximum external quantum efficiency (EQEmax) of $17.0 \%$. Zheng et al. reported an analog of CzpPhTRZ that replaced the Czp donor with a phenoxazinephane (PXZp). ${ }^{4}$ The generated molecule, PXZp-Ph-TRZ, possesses a red-shifted emission and a smaller $\Delta E_{\mathrm{ST}}$ of 0.03 eV leading to yellow TADF OLEDs with EQEmax of 7.8\%. Very recently, we designed and synthesized two deep blue TADF

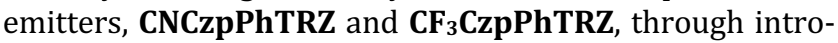
duction of electron-withdrawing cyano (CN) and trifluoromethyl $\left(\mathrm{CF}_{3}\right)$ groups onto the Czp moiety. CNCzpPhTRZ and CF $_{3}$ CzpPhTRZ emit at 458 and $456 \mathrm{~nm}$ with $\Phi_{\mathrm{PL}}$ of $65 \%$ and $70 \%$ in $10 \mathrm{wt} \%$ PPT doped film, respectively. ${ }^{5}$ Blue OLEDs exhibited EQE $\max$ of $7.4 \%$ at $456 \mathrm{~nm}$ for CNCzpPhTRZ, and EQEmax of $11.6 \%$ at $460 \mathrm{~nm}$ for $\mathbf{C F}_{3}$ CzpPhTRZ. As shown in Figure 1, only a single deck of the PCP has been elaborated in each of these TADF emitters.

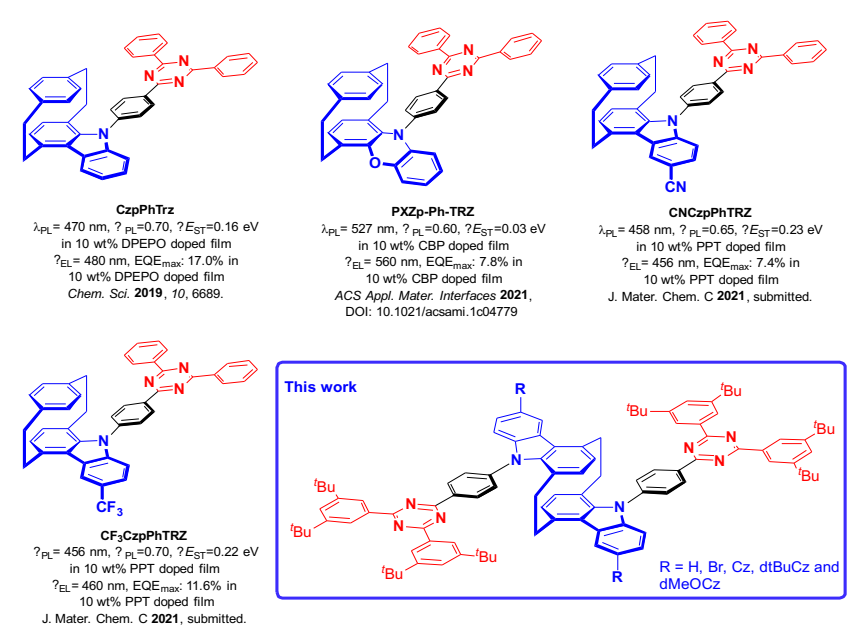

Figure 1. Chemical structures and performances of PCPbased emitters.

Here, we report the development of a new centrosymmetric through-space dicarbazolophane (DCCP) core, obtained through elaboration of both decks of the PCP. For the key building block, DCCP, we optimized a multi-gram scale efficient synthesis through a two-step protocol employing two-fold Pd-catalysed Buchwald-Hartwig amination afforded the $\mathrm{C}-\mathrm{N}$ coupling product 1 with a yield of $76 \%$ (Scheme 1). For the synthesis of the ring cyclization product, using $\mathrm{Pd}_{2}(\mathrm{dba})_{3}$ in combination with X-Phos as a catalyst system proved to be more effective for the two-fold Pdcatalysed oxidative cyclization employing the chlorinated moieties as synthetic handle. ${ }^{6}$ DCCP (2) was obtained in a yield of $49 \%$ (2.1 g scale). DCCP was regio- and chemoselectively dibrominated using NBS in THF to afford DCCP-2Br (3) in 68\% yield. Nucleophilic aromatic substitution with 2,4-bis(3,5-di-tert-butylphenyl)-6-(4-fluorophenyl)-1,3,5triazine produced DCCPTRZtBu 2 and the bromo-functionalized intermediate product $\mathbf{4}$ was further elaborated by grafting on 


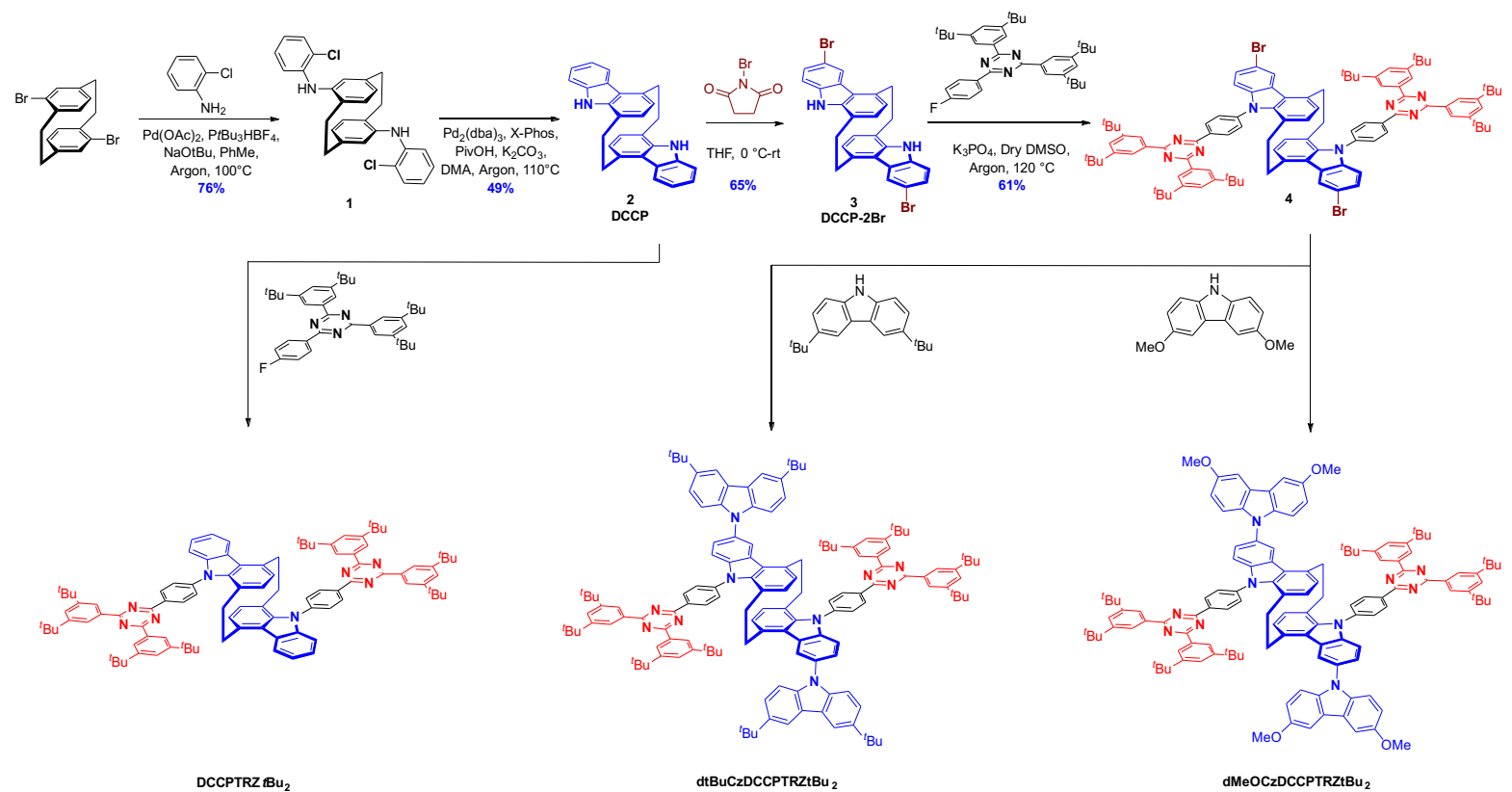

Scheme 1. Synthetic design towards dicarbazolophane-based emitters (details see ESI).

peripheral donors 3,6-di-tert-butyl-9H-carbazole (dtBuCz) and 3,6-dimethoxy-9H-carbazole ( $\mathrm{dMeOCz}$ ) via a two-fold Pd-catalyzed Buchwald-Hartwig cross-coupling to afford

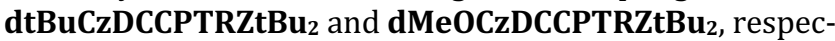
tively. These emitters were fully characterized by NMR spectroscopy, mass spectrometry, IR spectroscopy, and Elemental analysis (EA, details see ESI).

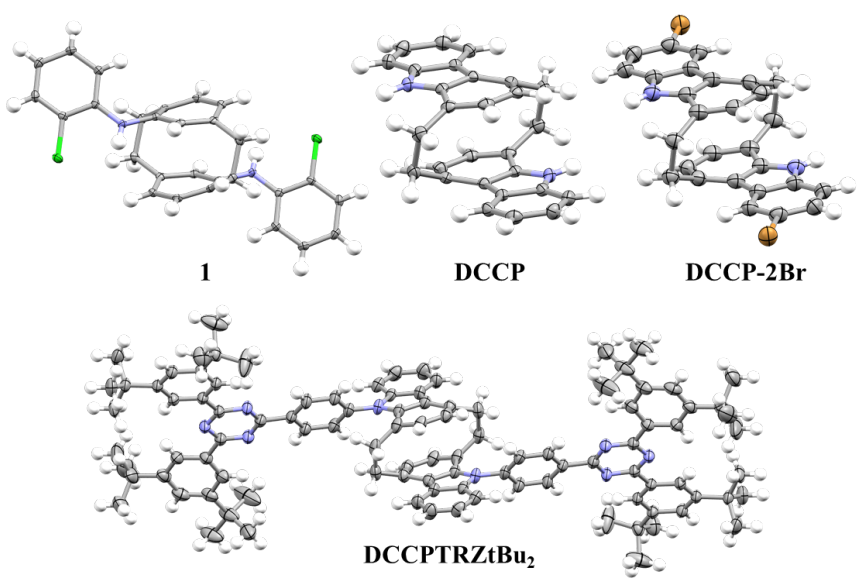

Figure 2. Thermal ellipsoid plots of the crystal structure of 1, DCCP, DCCP-2Br, and DCCPTRZtBu 2 . Ellipsoids are plotted at the $50 \%$ probability level.

The molecular structure of the compounds 1, DCCP, DCCP-2Br, and DCCPTRZtBu 2 were confirmed by single crystal X-ray analysis (Figure 2). The distances between the two benzene decks of the PCP are $3.01 \AA$ for $\mathbf{1}, 3.03 \AA$ for DCCP, $3.02 \AA$ A for DCCP-2Br, and $3.07 \AA$ for DCCPTRZtBu2, which are somewhat reduced compared to that in the parent PCP $(3.09 \AA)$.

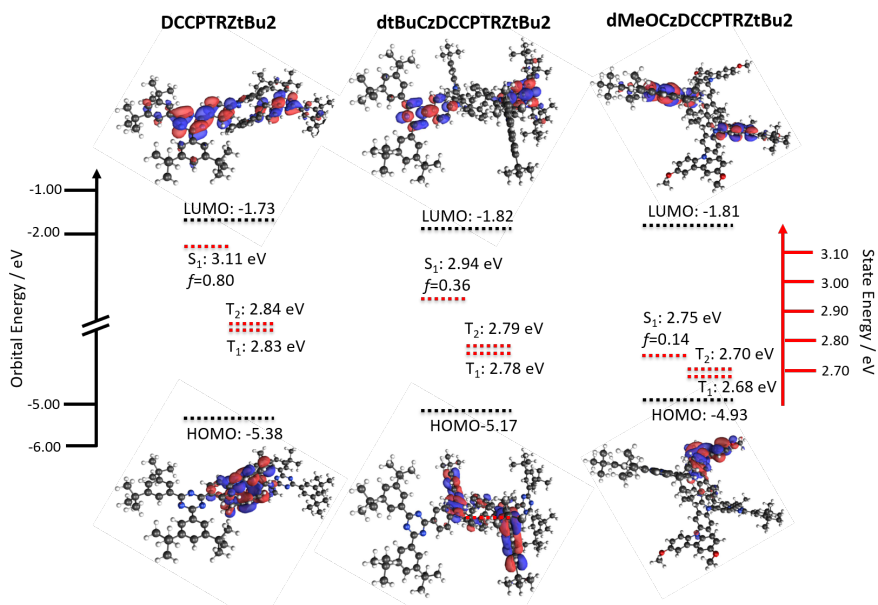

Figure 3. HOMO and LUMO electron density distribution, energy levels of excited states and oscillator strengths for the $\mathrm{S}_{1}-\mathrm{S}_{0}$ transitions of the DCCP-based emitters.

Theoretical calculations were employed to establish whether the DCCP-based emitters are likely to show TADF (Figure 3). The previously reported CzpPhTrz has a calculated HOMO level of $-5.54 \mathrm{eV}$, and a $\Delta E_{S T}$ of $0.30 \mathrm{eV}$. DCCPTRZtBu 2 presents a slightly smaller $\Delta E_{S T}$ of $0.28 \mathrm{eV}$ and a HOMO level that is destabilized at $-5.38 \mathrm{eV}$. The extended conjugation present in the DCCP compound results in a stronger donor character that is reflected in the shallower HOMO level. The addition of secondary substituted carbazole groups act to further increase the strength of the donor, which leads to a further destabilization of the HOMO level to $-5.17 \mathrm{eV}$ for dtBuCzDCCPTRZtBu 2 , and $-4.93 \mathrm{eV}$ for dMeOCzDCCPTRZtBuz. The singlet energy level is strongly affected by the strength of the donor, as we observe a significant decrease in its energy from $3.11 \mathrm{eV}$ to $2.94 \mathrm{eV}$, to $2.75 \mathrm{eV}$ with increasing strength of the donors present in 
Table 1. Photophysical properties of emitters.

\begin{tabular}{|c|c|c|c|c|c|c|c|c|c|}
\hline \multirow[t]{2}{*}{ Compound } & \multicolumn{4}{|c|}{ Toluene } & \multicolumn{5}{|c|}{ PVK (10 wt\%) thin film } \\
\hline & $\begin{array}{l}\lambda_{\mathrm{PL}} / \\
\mathrm{nm}\end{array}$ & $\Phi_{\mathrm{PL}^{a}} / \%$ & $\begin{array}{l}\tau_{\mathrm{p}} / \\
\mathrm{ns}\end{array}$ & $\begin{array}{l}\Delta E_{\mathrm{ST}} / \\
\mathrm{meV}\end{array}$ & $\begin{array}{l}\lambda_{\mathrm{PL}} / \\
\mathrm{nm}\end{array}$ & $\Phi_{\mathrm{PL}^{b}} / \%$ & $\begin{array}{l}\tau_{\mathrm{p}^{c}} / \\
\mathrm{ns}\end{array}$ & $\tau_{\mathrm{d}} / \mu \mathrm{s}$ & $\begin{array}{l}\Delta E_{\mathrm{ST}} / \\
\mathrm{meV}\end{array}$ \\
\hline DCCPTRZtBu 2 & 443 & $91(84)$ & 16 & 330 & 455 & $41(40)$ & 10 & -- & 115 \\
\hline dtBuCzDCCPTRZtBu2 & 458 & $89(89)$ & 21 & 290 & 455 & $37(35)$ & 7.4 & 9.7 & 110 \\
\hline dMeOCzDCCPTRZtBu & 475 & $44(31)$ & 29 & 230 & 490 & $41(38)$ & 6.8 & 7.7 & 70 \\
\hline
\end{tabular}

${ }^{a}$ Quinine sulfate $(0.5 \mathrm{M})$ in $\mathrm{H}_{2} \mathrm{SO}_{4}(\mathrm{aq})$ was used as the reference $\left(\Phi_{\mathrm{PL}}=54.6 \%, \lambda_{\mathrm{exc}}=360 \mathrm{~nm}\right)$. Values are given for degassed (aerated) solutions, ${ }^{b}$ purged with nitrogen (oxygen), ${ }^{c}$ average lifetime calculated as $\tau_{\text {avg }}=\left(\sum A_{i} \tau_{i}\right)\left(\sum \mathrm{A}_{i}\right)^{-1}$.

dtBuCzDCCPTRZtBuz and dMeOCzDCCPTRZtBuz. The triplet energy level also decreases in energy with increasing donor strength, but not as dramatically as the singlet. This leads to a decrease in $\Delta E_{\mathrm{ST}}$ of $0.15 \mathrm{eV}$ and $0.08 \mathrm{eV}$, respectively. All three materials present an intermediate triplet state that is slightly destabilized with respect to the $\mathrm{T}_{1}$ state. As a result, the increased density of triplet states should lead to an enhancement of the RISC rates. ${ }^{7}$

The energy levels of the emitters were inferred from an analysis of the oxidation and reduction potentials determined by cyclic voltammetry (CV) and differential pulse voltammetry (DPV) in dichloromethane (DCM) (Figure S22 and Table S28). For DCCPTRZtBu 2 and dtBuCzDCCPTRZ$\mathbf{t B u}_{2}$ the oxidation was found to be irreversible at a potential of $1.19 \mathrm{~V}$ vs. SCE and $1.09 \mathrm{~V}$ vs. SCE, respectively. The oxidation potential for DCCPTRZtBu 2 is significantly destabilized compared to that of CzpPhTRZ at $1.35 \mathrm{~V}$ (the original value reported was $1.14 \mathrm{~V}$; however, this has been revised upon re-examination of the voltammogram), thus, in line with the theoretical calculations. dMeOCzDCCPTRZ$\mathbf{t B u}_{2}$ exhibits a reversible oxidation at $0.90 \mathrm{~V}$ vs. SCE. The substitutions at the carbazoles in the donor moiety of dtBuCzDCCPTRZtBu 2 and dMeOCzDCCPTRZtBu lead to $_{2}$ a destabilization of the HOMOs, resulting in the expected cathodic shift of oxidation peak potentials. This observation is confirmed by the DFT calculations. All compounds show irreversible reduction waves with a peak potential of $-1.62 \mathrm{~V}$, -1.78 V, and -1.72 V for DCCPTRZtBu 2 , dtBuCzDCCPTRZtBu 2 , and dMeOCzDCCPTRZtBu2, respectively.

The absorption spectra (Figure S23), steady-state, and time-resolved photoluminescence (PL) are shown in Figure 4 and the data summarised in Table 1. The emission spectrum in toluene is red-shifted with increasing donor strength from DCCPTRZtBu 2 at $\lambda_{\mathrm{PL}}=443 \mathrm{~nm}$ to $\lambda_{\mathrm{PL}}=$ $458 \mathrm{~nm}$ for dtBuCzDCCPTRZtBu 2 and $\lambda_{\mathrm{PL}}=475 \mathrm{~nm}$ for dMeOCzDCCPTRZtBu2. The PL quantum yield ( $\left.\Phi_{\mathrm{PL}}\right)$ of degassed toluene solutions of DCCPTRZ and dtBuCzDCCPTRZtBu 2 are $91 \%$ and $89 \%$, respectively, and showed no significant change upon exposure to oxygen, which suggests the intersystem crossing between $\mathrm{S}_{1}$ and $\mathrm{T}_{1}$ is negligible. ${ }^{8}$ The transient PL of DCCPTRZtBu2, dtBuCzDCCPTRZtBu ${ }_{2}$ and dMeOCzDCCPTRZtBu 2 were found to decay monoexponentially, with lifetimes of $16 \mathrm{~ns}$, $21 \mathrm{~ns}$, and 29 ns, respectively; therefore, in toluene these three compounds are fluorescent. For all compounds the $\Delta E_{\text {ST }}$ in toluene was measured to be $>200 \mathrm{meV}$ (Figure S26) and therefore unlikely to support efficient reverse intersystem crossing (RISC) at room temperature. However, upon rapid cooling using liquid nitrogen, the emitters at 77 $\mathrm{K}$ would be frozen in their respective non-equilibrised geometries, resulting in an overestimation of $\Delta E_{\mathrm{ST}}$.

The compounds were then studied as doped poly $(N$-vinyl carbazole) (PVK) films at a concentration of $10 \mathrm{wt} \%$, the value of which was chosen to optimise the $\Phi_{P L}$ (Table 1). The steady-state PL spectra and transient PL decays of the compounds are shown in Figure 4. The steady-state PL spectra show the same trends as those in solution but are red-shifted with $\lambda_{\mathrm{PL}}=455 \mathrm{~nm}$ for $\mathbf{D C C P T R Z t B u} 2, \lambda_{\mathrm{PL}}=$ $455 \mathrm{~nm}$ for dtBuCzDCCPTRZtBu 2 , and $\lambda_{\mathrm{PL}}=490 \mathrm{~nm}$ for dMeOCzDCCPTRZtBu2. The $\Phi_{\mathrm{PL}}$ for the films is around $40 \%$, which is significantly lower than in solution. This may be due host-emitter or emitter-emitter deactivation in the films. The transient PL decays of all films show a multiexponential prompt emission with an average lifetime, $\tau_{\mathrm{p}}$, of $10 \mathrm{~ns}, \quad 7.4 \mathrm{~ns}$, and $6.8 \mathrm{~ns}$ for DCCPTRZtBu 2 , dtBuCzDCCPTRZtBu, and dMeOCzDCCPTRZtBu, respectively. The transient PL of dtBuCzDCCPTRZtBu 2 , and dMeOCzDCCPTRZtBu2 show a multiexponential delayed lifetime component with an average lifetime, $\tau_{d}$, of $9.7 \mu \mathrm{s}$ and $7.7 \mu \mathrm{s}$ at $300 \mathrm{~K}$. The delayed emission lifetime is comparable to the delayed component of CzpPhTrz in PVK of $9.0 \mu$ s (Figure S25).
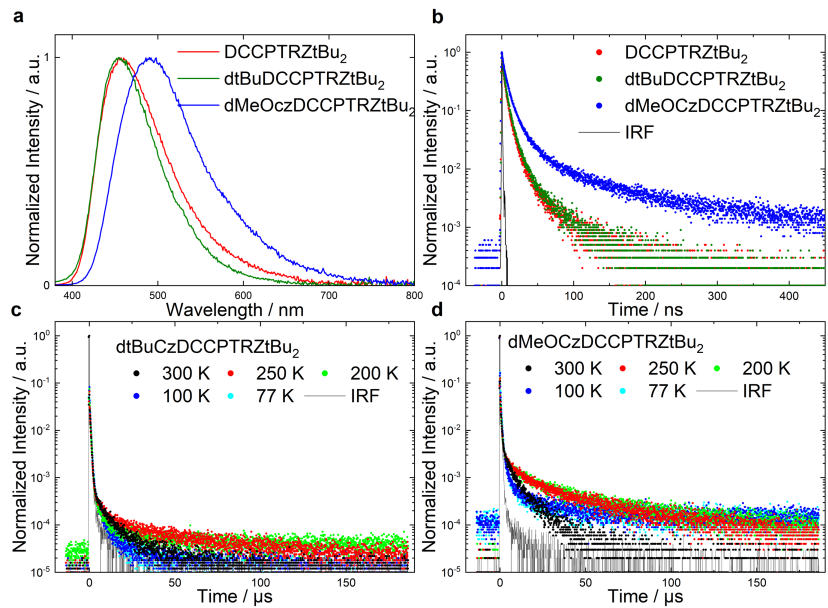

Figure 4. Photoluminescence of emitters in PVK film (10 wt\%). (a) Steady-state PL spectra $\left(\lambda_{\text {exc }}=345 \mathrm{~nm}\right)$, (b) prompt PL decay of all emitters, (c) delayed PL decay component at different temperatures of dtBuCzDCCPTRZtBu 2 and (d) dMeOCzDCCPTRZtBu2. For transient PL $\left(\lambda_{\mathrm{exc}}=\right.$ $378 \mathrm{~nm})$. 
The delayed component is longer-lived at lower temperatures and does not show a complete quenching at low temperature as expected for a TADF emitter. The $\Delta E_{\mathrm{ST}}$ values are $110 \mathrm{meV}$ and $70 \mathrm{meV}$ for dtBuCzDCCPTRZtBuz, and dMeOCzDCCPTRZtBu , $_{2}$ respectively (Figure S27), and thus sufficiently small for TADF to be operational at room temperature. The short delayed lifetime and moderate $\Phi_{\mathrm{PL}}$ at room temperature can be explained by a strong non-radiative decay contribution from the triplet state. At lower temperatures this pathway is quenched due to fewer vibrations resulting in a longer delayed lifetime. In the case of dMeOCzDCCPTRZtBu 2 , RISC does not appear to be fully suppressed at $77 \mathrm{~K}$. Assuming the previous discussed measured $\Delta E_{\mathrm{ST}}=70 \mathrm{meV}$ is identical to the energy barrier for RISC in dMeOCzDCCPTRZtBu $\mathbf{d}_{2}$, we expect $k_{\text {RISC }}$ to be reduced by four orders of magnitude at $77 \mathrm{~K}$ from $k_{\mathrm{RISC}}$ at $300 \mathrm{~K}$ but not entirely quenched, resulting in a long delayed emission lifetime.

Given the high molecular weights of the emitters, solution-processed devices were fabricated with the following layers: indium tin oxide (ITO) / poly(3,4-ethylenedioxythiophene) polystyrene sulfonate (PEDOT:PSS) (40 nm)/emitter:PVK (10 wt\%, 35-40 nm) / 1,3,5-tris(3-pyridyl-3-phenyl)benzene (TmPyPB) (50 nm)/LiF (0.5 nm)/Al (100 nm). The electroluminescence properties are shown in Figure S28 and the device metrics are listed in Table S29. Analogous to the trend observed in the PL study, the emission colour of the devices progressively red-shifts from sky-blue emission for DCCPTRZtBu2 with a $\lambda_{\mathrm{EL}}$ of $475 \mathrm{~nm}$, to $\lambda_{\mathrm{EL}}=478 \mathrm{~nm}$ for $\mathbf{d t B u C z D C C P T R Z t B u} 2$ to $\lambda_{\mathrm{EL}}=515 \mathrm{~nm}$ for dMeOCzDCCPTRZtBu . The EL spectra are greener (i.e. relatively red-shifted) than the corresponding PL spectra in the PVK host at the same doping concentration. This might be due to microcavity effects in the device.

The highest $E Q E_{\max }$ was found for the device with dMeOCzDCCPTRZtBu$_{2}$ at $8.2 \%$, and this OLED achieved an efficiency of $7.9 \%$ at $100 \mathrm{~cd} / \mathrm{m}^{2}$. Devices with DCCPTRZtBu 2 and dtBuCzDCCPTRZtBuz exhibited much lower EQE$\max$ of $3.2 \%$ and $4.0 \%$, respectively. Based on the previous discussed PLQY of the PVK films doped with dMeOCzDCCPTRZtBu2 $\left(\Phi_{\mathrm{PL}}=41 \%\right)$ the theoretical EQEmax is $8.2 \%$ when considering an outcoupling efficiency of $\chi_{\text {out }} \approx 20 \%$, and that all triplet excitons are efficiently converted into singlets. Therefore, we conclude that the OLED operates via an efficient TADF mechanism. The lower efficiency in devices with either DCCPTRZtBu 2 or

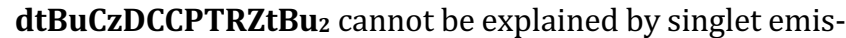
sion alone as this would lead to an expected $\mathrm{EQE}_{\max }$ of $2.1 \%$ and $1.9 \%$, respectively. Therefore, triplet up conversion must occur in these devices too; however, with a lower exciton utilization efficiency than in dMeOCzDCCPTRZtBu 2 , which is likely due to their larger $\Delta E_{\mathrm{ST}}$. DCCPTRZtBu 2 did not show any indication of TADF in doped films within our PL studies. As the EQEmax is observed at higher current densities as in the devices with dMeOCzDCCPTRZtBu 2 , the upconversion might be explained by triplet-triplet annihilation, which is facilitated by a large triplet population.

In this work, we describe the design and modular synthesis of centrosymmetric DCCP-based through-space donors via a multi-fold stepwise Pd-catalyzed Buchwald-Hartwig amination and ring cyclization approach. The derived emitters, DCCPTRZtBu2, dtBuCzDCCPTRZtBu2, and
dMe0CzDCCPTRZtBu 2 showed $\Phi_{\mathrm{PL}}$ of up to $91 \%$ in toluene and $41 \%$ in doped PVK films. Consequently, solution-processed OLEDs using dMeOCzDCCPTRZtBu2 were fabricated and achieved an EQE $E_{\max }$ of $8.2 \%$ via an efficient TADF mechanism.

\section{ASSOCIATED CONTENT}

\section{Supporting Information}

The Supporting Information is available free of charge on the ACS Publications website.

Preparation, X-ray crystallography data (1, CCDC 2049645; DCCP (2), CCDC 2049646; DCCP-2Br (3), CCDC 2049647; and DCCPTRZtBu 2, CCDC 2049648), photophysical properties, calculation details, NMR spectra.

\section{AUTHOR INFORMATION}

\section{Corresponding Authors}

Stefan Bräse - Institute of Organic Chemistry, Karlsruhe Institute of Technology (KIT), Fritz-Haber-Weg 6, 76131 Karlsruhe, Germany. E-mail: braese@kit.edu

Eli Zysman-Colman - Organic Semiconductor Centre, EaStCHEM School of Chemistry, University of St Andrews, St Andrews, Fife, KY16 9ST, UK. E-mail: eli.zysman-colman @standrews.ac.uk

Ifor D. W. Samuel - Organic Semiconductor Centre, SUPA, School of Physics and Astronomy, University of St Andrews, North Haugh, St Andrews, KY16 9SS, UK. E-mail: idws@st-andrews.ac.uk

\footnotetext{
Authors

Zhen Zhang - Institute of Organic Chemistry, Karlsruhe Institute of Technology (KIT), Fritz-Haber-Weg 6, 76131 Karlsruhe, Germany.

Stefan Diesing - Organic Semiconductor Centre, EaStCHEM School of Chemistry, University of St Andrews, St Andrews, Fife, KY16 9ST, UK.

Ettore Crovini - Organic Semiconductor Centre, EaStCHEM School of Chemistry, University of St Andrews, St Andrews, Fife, KY16 9ST, UK.

Abhishek Kumar Gupta - Organic Semiconductor Centre, EaStCHEM School of Chemistry, University of St Andrews, St Andrews, Fife, KY16 9ST, UK.

Eduard Spuling - Institute of Organic Chemistry, Karlsruhe Institute of Technology (KIT), Fritz-Haber-Weg 6, 76131 Karlsruhe, Germany.

Xuemin Gan - Institute of Organic Chemistry, Karlsruhe Institute of Technology (KIT), Fritz-Haber-Weg 6, 76131 Karlsruhe, Germany.

Olaf Fuhr - Institute of Nanotechnology (INT) and Karlsruhe Nano-Micro Facility (KNMF), Karlsruhe Institute of Technology (KIT), Hermann-von-Helmholtz-Platz 1, 76344 Eggenstein-Leopoldshafen, Germany

Martin Nieger - Department of Chemistry, University of Helsinki, P.O. Box 55 A.I. Virtasen aukio 1, 00014 University of Helsinki,

Finland

Zahid Hassan - Institute of Organic Chemistry, Karlsruhe Institute of Technology (KIT), Fritz-Haber-Weg 6, 76131 Karlsruhe, Germany.
}

Zhen Zhang $\ddagger$ and Stefan Diesing ${ }^{\ddagger}$ contributed equally to this work. 


\section{Notes}

The authors declare no competing financial interest.

\section{ACKNOWLEDGMENT}

The German Research Foundation (formally Deutsche Forschungsgemeinschaft DFG) in the framework of SFB1176 Cooperative Research Centre "Molecular Structuring of Soft Matter" (CRC1176, A4, B3, C2, C6) and the cluster "3D Matter Made to Order" all funded under Germany's Excellence Strategy 2082/1-390761711 are acknowledged for financial contributions. A. K. G. is thankful to the Royal Society for a Newton International Fellowship NF171163. E. Z.-C. and I. D. W. S. acknowledge support from EPSRC (EP/L017008, EP/P010482/1, EP/R035164/1). E. C. and E. Z.-C. acknowledge the EU Horizon 2020 grant agreement no. 812872 (TADFlife).

\section{REFERENCES}

(1). (a) Cram, D. J.; Cram, J. M. Cyclophane Chemistry: Bent and Battered Benzene Rings. Acc. Chem. Res. 1971, 4, 204-213. (b) Zyss, J.; Ledoux, I.; Volkov, S.; Chernyak, V.; Mukamel, S.; Bartholomew, G. P.; Bazan, G. C. Through-Space Charge Transfer and Nonlinear Optical Properties of Substituted Paracyclophane. J. Am. Chem. Soc. 2000, 122, 11956-11962. (c) Hong, J. W.; Woo, H. Y.; Liu, B.; Bazan, G. C. Solvatochromism of Distyrylbenzene Pairs Bound Together by [2.2]Paracyclophane: Evidence for a Polarizable "Through-Space" Delocalized State. J. Am. Chem. Soc. 2005, 127, 7435-7443. (d) Morisaki, Y.; Chujo, Y. Through-space conjugated polymers consisting of [2.2] paracyclophane. Polym. Chem. 2011, 2, 12491257. (e) Morisaki, Y.; Chujo, Y. $\pi$-Electron-system-layered polymers based on [2.2] paracyclophane. Chem. Lett. 2012, 41, 840846. (f) Morisaki, Y.; Gon, M.; Sasamori, T.; Tokitoh, N.; Chujo, Y. Planar chiral tetrasubstituted [2.2]paracyclophane: optical resolution and functionalization. J. Am. Chem. Soc. 2014, 136, 3350-3353. (g) Gon, M.; Morisaki, Y.; Chujo, Y. A silver(i)-induced higher-ordered structure based on planar chiral tetrasubstituted [2.2] paracyclophane. Chem. Commun. 2017, 53, 8304-8307. (h) Hassan, Z.; Spuling, E.; Knoll, D. M.; Lahann, J.; Bräse, S. Planar chiral [2.2]paracyclophanes: from synthetic curiosity to applications in asymmetric synthesis and materials. Chem. Soc. Rev. 2018, 47, 6947-6963. (i) Zhang, M. Y.; Li, Z. Y.; Lu, B.; Wang, Y.; Ma, Y. D.; Zhao, C. H. Solid-state emissive triarylborane-based [2.2]paracyclophanes displaying circularly polarized luminescence and thermally activated delayed fluorescence. Org. Lett. 2018, 20, 6868-6871. (j) Zhang, M. Y.; Liang, X.; Ni, D. N.; Liu, D. H.; Peng, Q.; Zhao, C. H. 2-(Dimesitylboryl)phenyl-substituted [2.2] paracyclophanes featuring intense and sign-invertible circularly polarized luminescence. Org. Lett. 2021, 23, 2-7.

(2). (a) Bartholomew, G. P.; Bazan, G. C. Bichromophoric Paracyclophanes: Models for Interchromophore Delocalization. Acc. Chem. Res. 2001, 34, 30-39. (b) Marrocchi, A.; Tomasi, I.; Vaccaro, L. Organic small molecules for photonics and electronics from the [2.2] paracyclophane scaffold. Isr. J. Chem. 2012, 52, 41-52. (c) Spuling, E.; Sharma, N.; Samuel, I. D. W.; Zysman-Colman, E.; Brase, S. (Deep) blue through-space conjugated TADF emitters based on [2.2] paracyclophanes. Chem. Commun. 2018, 54, 9278-9281. (d) Wang, C. S.; Wei, Y. C.; Chang, K. H.; Chou, P. T.; Wu, Y. T. Indeno[1,2b]fluorene-based [2,2]cyclophanes with $4 n / 4 n$ and $4 n /[4 n+2]$ pi electrons: syntheses, structural analyses, and excitonic coupling properties. Angew. Chem. Int. Ed. 2019, 58, 10158-10162. (e) Liang, X.; Liu, T. T.; Yan, Z. P.; Zhou, Y.; Su, J.; Luo, X. F.; Wu, Z. G.; Wang, Y.; Zheng, Y. X.; Zuo, J. L. Organic Room-Temperature Phosphorescence with Strong Circularly Polarized Luminescence Based on Paracyclophanes. Angew. Chem. Int. Ed. 2019, 58, 17220 17225. (f) Hassan, Z.; Spuling, E.; Knoll, D. M.; Brase, S. Regioselective Functionalization of [2.2]Paracyclophanes: Recent Synthetic
Progress and Perspectives. Angew. Chem. Int. Ed. 2020, 59, 21562170 .

(3). Sharma, N.; Spuling, E.; Mattern, C. M.; Li, W.; Fuhr, O.; Tsuchiya, Y.; Adachi, C.; Brase, S.; Samuel, I. D. W.; Zysman-Colman, E. Turn on of sky-blue thermally activated delayed fluorescence and circularly polarized luminescence (CPL) via increased torsion by a bulky carbazolophane donor. Chem. Sci. 2019, 10, 6689-6696.

(4). Liao, C.; Zhang, Y.; Ye, S. H.; Zheng, W. H. Planar Chiral [2.2]Paracyclophane-Based Thermally Activated Delayed Fluorescent Materials for Circularly Polarized Electroluminescence. ACS Appl. Mater. Interfaces 2021, 13, 25186-25192.

(5). Gupta, A. K.; Zhang, Z.; Spuling, E.; Kaczmarek, M.; Wang, Y.; Hassan, Z.; Samuel, I. D. W.; Bräse, S.; Zysman-Colman. E. Electronwithdrawing Group Modified Carbazolophane Donors for Deep Blue Thermally Activated Delayed Fluorescence OLEDs. J. Mater. Chem. C 2021, submitted.

(6). (a) Lennartz, P.; Raabe, G.; Bolm, C. Synthesis of Planar Chiral Carbazole Derivatives Bearing a [2.2]Paracyclophane Skeleton. Isr. J. Chem. 2012, 52, 171-179. (b) Buchwald, S. L.; Huang, W. [22]paracyclophane-derived donor/acceptor type molecular for OLED applications. WO2016196885 A1.

(7). (a). Santos, P. L.; Ward, J. S.; Data, P.; Batsanov, A. S.; Bryce, M. R.; Dias, F. B.; Monkman, A. P. Engineering the singlet-triplet energy splitting in a TADF molecule. J. Mater. Chem. C 2016, 4, 38153824. (b) Hosokai, T.; Matsuzaki, H.; Nakanotani, H.; Tokumaru, K.; Tsutsui, T.; Furube, A.; Nasu, K.; Nomura, H.; Yahiro, M.; Adachi, C. Evidence and mechanism of efficient thermally activated delayed fluorescence promoted by delocalized excited states. Sci. Adv. 2017, 3, e1603282. (c) Noda, H.; Nakanotani, H.; Adachi, C. Excited state engineering for efficient reverse intersystem crossing. Sci. $A d v$. 2018, 4, eaao6910. (d) Samanta, P. K.; Kim, D.; Coropceanu, V.; Bredas, J. L. Up-Conversion Intersystem Crossing Rates in Organic Emitters for Thermally Activated Delayed Fluorescence: Impact of the Nature of Singlet vs Triplet Excited States. J. Am. Chem. Soc. 2017, 139, 4042-4051.

(8). (a) Melhuish, W. H. Quantum Efficiencies of Fluorescence of Organic Substances : Effect of Solvent and Concentration of the Fluorescent solute. J. Phys. Chem. 1961, 65, 229-235. (b) Demas, J. N.; Crosby, G. A. The Measurement of Photoluminescence Quantum Yields. J. Phys. Chem. 1971, 75, 991-1024. 